Service social

\title{
Comprendre les masculinités rurales : un portrait basé sur la santé psychologique
}

\section{Philippe Roy et Gilles Tremblay}

Volume 58, numéro 1, 2012

URI : https://id.erudit.org/iderudit/1010442ar

DOI : https://doi.org/10.7202/1010442ar

Aller au sommaire du numéro

\section{Éditeur(s)}

École de service social de l’Université Laval

ISSN

1708-1734 (numérique)

Découvrir la revue

Citer cet article

Roy, P. \& Tremblay, G. (2012). Comprendre les masculinités rurales : un portrait basé sur la santé psychologique. Service social, 58(1), 68-84.

https://doi.org/10.7202/1010442ar
Résumé de l'article

Les hommes ruraux forment une clientèle particulièrement vulnérable en matière de suicide et de détresse psychologique et ils utilisent peu les services de santé. Comme toute inégalité sociale de santé, cette situation se heurte à plusieurs valeurs chères au service social. Malgré son portrait préoccupant, la santé psychologique des hommes ruraux demeure un sujet largement négligé au Québec, comme dans la plupart des pays industrialisés, ce qui contribue à son invisibilité. Cet article vise à mieux comprendre les réalités objectives et subjectives des hommes ruraux qui influencent leur santé psychologique. Un volet de l'analyse aborde les principaux paradigmes compréhensifs des hommes et des masculinités qui se sont intéressées aux masculinités rurales, ce qui ouvre sur des pistes de recherche. 


\title{
Comprendre les masculinités rurales : un portrait basé sur la santé psychologique
}

\author{
Philippe Roy* \\ Gilles Tremblay
}

\section{RÉSUMÉ}

Les hommes ruraux forment une clientèle particulièrement vulnérable en matière de suicide et de détresse psychologique et ils utilisent peu les services de santé. Comme toute inégalité sociale de santé, cette situation se heurte plusieurs valeurs chères au service social. Malgré son portrait préoccupant, la santé psychologique des hommes ruraux demeure un sujet largement négligé au Québec, comme dans la plupart des pays industrialisés, ce qui contribue à son invisibilité. Cet article vise à mieux comprendre les réalités objectives et subjectives des hommes ruraux qui influencent leur santé psychologique. Un volet de l'analyse aborde les principaux paradigmes compréhensifs des hommes et des masculinités qui se sont intéressées aux masculinités rurales, ce qui ouvre sur des pistes de recherche.

Mots-clés : rural, masculinités, genre, dépression, suicide, détresse psychologique

\begin{abstract}
Rural men form a group particularly vulnerable to health problems such as suicide and psychological distress and rarely use health services. Like any social health inequality, this situation is a challenge regarding many values of social work. Despite the worrying portrait, rural men's psychological health remains largely neglected in Québec, like in most of the industrialized countries, which contributes to its invisibility. This paper aims to a better understanding of the objective and subjective realities that influence their psychological health. It presents the main paradigms used to understand rural masculinities and suggests future directions for research.
\end{abstract}

Keywords: rural, masculinities, gender, depression, suicide, psychological distress

Au cours des trente dernières années, la recherche sur les hommes et les masculinités s'est grandement développée à travers le monde. Loin d'échapper à cette tendance, le Québec se démarque avantageusement sur le plan international par le dynamisme et le rayonnement de ses productions scientifiques. En combinant plusieurs sources de connaissance, nous constatons que les hommes ruraux forment une clientèle particulièrement vulnérable en matière de santé psychologique. Comme toute inégalité sociale de santé, cette situation se heurte à

\footnotetext{
L'auteur principal reconnaît le soutien financier du Fonds québécois de recherche sur la société et la culture, l'équipe de recherche Masculinités et Société et la Fondation Desjardins.
} 
plusieurs valeurs chères au service social. Malgré son portrait préoccupant, la santé psychologique des hommes ruraux demeure un sujet largement négligé au Québec, comme dans la plupart des pays industrialisés, ce qui contribue à son invisibilité (Pugh et Cheers, 2010).

Cet article repose sur une recension des écrits réalisée à partir des bases de données électroniques en utilisant les mots clés: agriculteurs (farmers), rural, hommes (men), masculinité-s (masculinity-ies), dépression, suicide, adaptation (coping), résilience. Cinquantecinq (55) publications ont été retenues sur la base de leur pertinence pour mieux comprendre les réalités objectives et subjectives des hommes ruraux qui influencent leur portrait de santé psychologique. De ce nombre, 25 sont des études empiriques (17 quantitatives, 5 qualitatives et 3 mixtes), 22 des articles ou chapitres théoriques et huit (8) sont des articles ou chapitres cliniques. Sur le plan géographique, les publications proviennent du Québec (14), des ÉtatsUnis (14), du Canada (9), de l'Australie (8), de l'Europe (6) et quatre (4) publications proviennent d'un collectif international.

En premier lieu, il importe de définir la masculinité et la ruralité. Par la suite, l'état des connaissances sur la problématique se décline en deux volets: le portrait de santé psychologique des hommes ruraux et leur marginalisation. Troisièmement, nous analyserons les principaux paradigmes compréhensifs des hommes et des masculinités qui se sont intéressées aux masculinités rurales, ce qui ouvre sur des pistes de recherche.

\section{MASCULINITÉ ET RURALITÉ}

Cet article s'inscrit dans la lignée des études de genre, soit la différenciation sociale et non biologique qui est faite entre les hommes et les femmes. On entend par « réalités masculines » les expériences subjectives et objectives vécues par les hommes (Lindsay, Rondeau et Desgagnés, 2010). Dans ce domaine d'étude, la masculinité est considérée comme «socialement constituée d'une combinaison de facteurs biologiques, de variables psychologiques et d'expériences sociales » (Levant et Habben, 2003, p. 176). La définition de ce qui est masculin peut varier pour différents groupes d'hommes, mais elle peut aussi varier chez un même individu selon la situation. C'est pourquoi il est plus approprié de parler des masculinités (Connell, 1995). Le genre est agi lorsque les comportements et les attitudes constituent des manières de démontrer son adhésion aux stéréotypes masculins ou sa distance par rapport à ces stéréotypes (Butler, 2005). Sur le plan de la santé par exemple, Robertson (2007) suggère que certains hommes expriment leur masculinité en négligeant leur santé (shouldn't care), entre autres quand ils évitent les visites chez le médecin ou qu'ils endurent la douleur jusqu'à la crise, alors que d'autres en prennent un grand soin (should care).

L'autre concept à la base de cette réflexion est celui de ruralité, dont la définition ne fait pas consensus (Fraser et al., 2002; Pugh et Cheers, 2010). Dans le sens commun, la ruralité est définie en opposition aux espaces urbains ou métropolitains. Les enquêtes populationnelles (Institut canadien d'information sur la santé, 2006; Martinez, Pampalon, Hamel et Raymond, 2004) définissent la ruralité selon la densité et la distance par rapport à la zone d'influence métropolitaine (ZIM). Mais cette définition est limitée puisqu'elle ne permet pas de traiter en 
profondeur de la représentation sociale de la ruralité (ICIS, 2006). Le lieu du domicile ne peut donc être le seul critère pour la définir.

Plusieurs travaux se sont penchés sur les valeurs distinctives des milieux ruraux (Collier, 2006; Farley, 1982; Lohmann et Lohmann, 2005; Martinez-Brawley, 1999). Ainsi, ces derniers sont caractérisés par un fort sens d'identité régionale. En outre, les vertus traditionnelles de travail, d'autonomie et d'autosuffisance sont valorisées à travers des liens familiaux « tissés serrés ». Le mode de vie rural est aussi fortement lié à l'exploitation des ressources naturelles et est rythmé par les saisons, d'où l'importance d'avoir une bonne connaissance des phénomènes naturels. Par ailleurs, dans les villages et petites municipalités où tout le monde se connaît, la méfiance peut être plus grande envers les étrangers, tout comme l'intimité plus difficile à préserver.

\section{RURALITÉ ET SERVICE SOCIAL}

Pugh et Cheers (2010, p. xvi) identifient certaines particularités du milieu rural qui ont une influence sur la pratique du travail social dans ce milieu, sans pour autant assumer qu'elles sont universelles :

1. L'expérience et les besoins de certains résidents ruraux tendent à être méconnus de la part des milieux de la recherche et de la formation en intervention sociale;

2. Les populations rurales sont typiquement défavorisées en matière d'accès aux soins de santé et de services sociaux;

3. Les infrastructures rurales tendent à être faibles (par exemple: les systèmes de transport en commun);

4. Les possibilités d'emploi sont limitées en raison des distances ainsi que des changements qu'a connus l'économie rurale;

5. La pauvreté est plus fréquente dans la plupart des communautés rurales;

6. Les services coûtent généralement plus cher à offrir en milieu rural qu'en milieu urbain.

Certaines études portent sur les populations rurales tandis que d'autres ciblent spécifiquement les agriculteurs. Une attention particulière doit être apportée à ce qui unit et ce qui distingue ces deux groupes (Miller et Burns, 2008). Au Québec, environ une personne sur cinq vit en milieu rural (Martinez et al., 2004), alors que la population agricole représente 5,7\% de l'ensemble de la population rurale (Statistique Canada, 2006).

Ce portrait du contexte rural constitue une piste de compréhension qui, jumelée à d'autres connaissances approfondies en matière de socialisation masculine, de service social et de santé mentale, permet un éclairage nouveau sur la santé psychologique des hommes ruraux. 


\section{LES PROBLÈMES DE SANTÉ PSYCHOLOGIQUE DES HOMMES RURAUX : UN POINT DE VULNÉRABILITÉ}

Les taux élevés de suicide que l'on retrouve chez les hommes ruraux font émerger la préoccupation pour le bien-être psychologique de ce groupe (ICIS, 2006; Kutek, Turnbull et Fairweather-Schmidt, 2011). À travers le monde, les taux de suicide des hommes ruraux tendent à être de trois à cinq fois plus élevés que les taux nationaux (Courtenay, 2011). Cette surmortalité importante révèle un groupe à risque élevé de suicide. Le Québec ne fait pas exception à cette tendance. Une étude épidémiologique de Martinez et al. (2004) révèle que, sur 100000 habitants, les hommes ruraux affichent un taux de suicide de 27 décès, comparativement à 20 décès chez les hommes urbains. Du côté des femmes, ces taux sont de 6,1 en milieu rural contre 5,7 en milieu urbain (voir Figure 1). L'écart entre populations urbaines et rurales est donc plus grand chez les hommes qu'il ne l'est chez les femmes.

Figure 1 : Taux de suicide par densité urbaine et par sexe, Québec (décès par 100000 habitants) (Martinez et al., 2004)

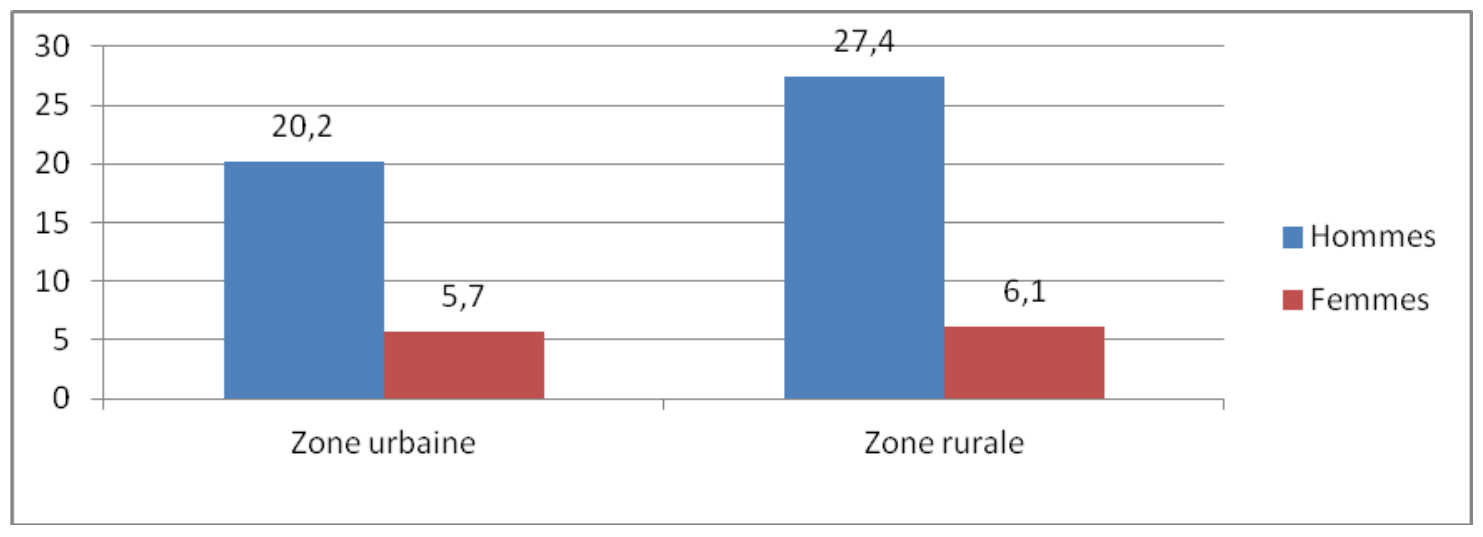

Les plus forts taux de suicide de la province se trouvent dans les régions principalement rurales comme la Gaspésie, le Bas-St-Laurent et la Mauricie. À l'inverse, ce sont les régions les plus densément peuplées (Montréal, Laval, Montérégie ${ }^{1}$ ) qui montrent les plus faibles taux de suicide (Gagné, Légaré, Perron et St-Laurent, 2011). Si le fort taux de suicide des hommes ruraux québécois a attiré peu d'attention jusqu'ici, la situation est bien différente lorsqu'il est question des agriculteurs.

Au cours des dernières années, plusieurs actions communautaires, événements et reportages médiatiques ont sonné l'alarme sur le suicide chez les agriculteurs. La seule étude disponible au Canada est celle de Pickett et al. (2000), basée sur une cohorte d'agriculteurs observée entre 1971 et 1986. Depuis cette cohorte, le monde de l'agriculture a connu beaucoup de transformations, ce qui soulève des questions quant à la validité actuelle de cette étude. Présentement, les statistiques québécoises ne permettent pas de connaître avec précision le taux de suicide chez les agriculteurs.

1. Même si la Montérégie comprend un important territoire rural, la majorité de sa population est concentrée dans les villes qui forment la Rive-Sud de Montréal, comme Longueuil. 
En revanche, ces données sont connues en Australie et en Angleterre, et elles révèlent que les agriculteurs ont des taux de suicide plus élevés que ceux de la population masculine générale ou ceux des autres hommes ruraux (Booth, Briscoe et Powell, 2000; Fraser et al., 2005; Judd, Jackson, Fraser et al., 2006; Judd, Jackson, Komiti et al., 2006).

Le suicide est souvent l'aboutissement d'un long processus multifactoriel qui comprend des problèmes de santé psychologique, dont la dépression, la détresse psychologique et les idéations suicidaires (Préville, Boyer, Hébert, Bravo et Seguin, 2005). En 2006, une importante enquête sur la santé psychologique des agriculteurs a été réalisée par Lafleur et Allard (2006). Cette enquête révélait que $50 \%$ d'entre eux (59\% des femmes et $49 \%$ des hommes) vivent un niveau élevé de détresse psychologique, comparativement à $20 \%$ dans le reste de la population (voir Figure 2). Cette proportion atteint même $66 \%$ chez les producteurs de porc. Les idéations suicidaires sont aussi plus fréquentes parmi la population agricole, hommes et femmes (6,6\%), que dans la population générale (3,9\%).

Figure 2 : Comparaison de la détresse psychologique élevée et des idéations suicidaires, population agricole (Lafleur et Allard, 2006) et générale (Camirand et Nahnou, 2008)

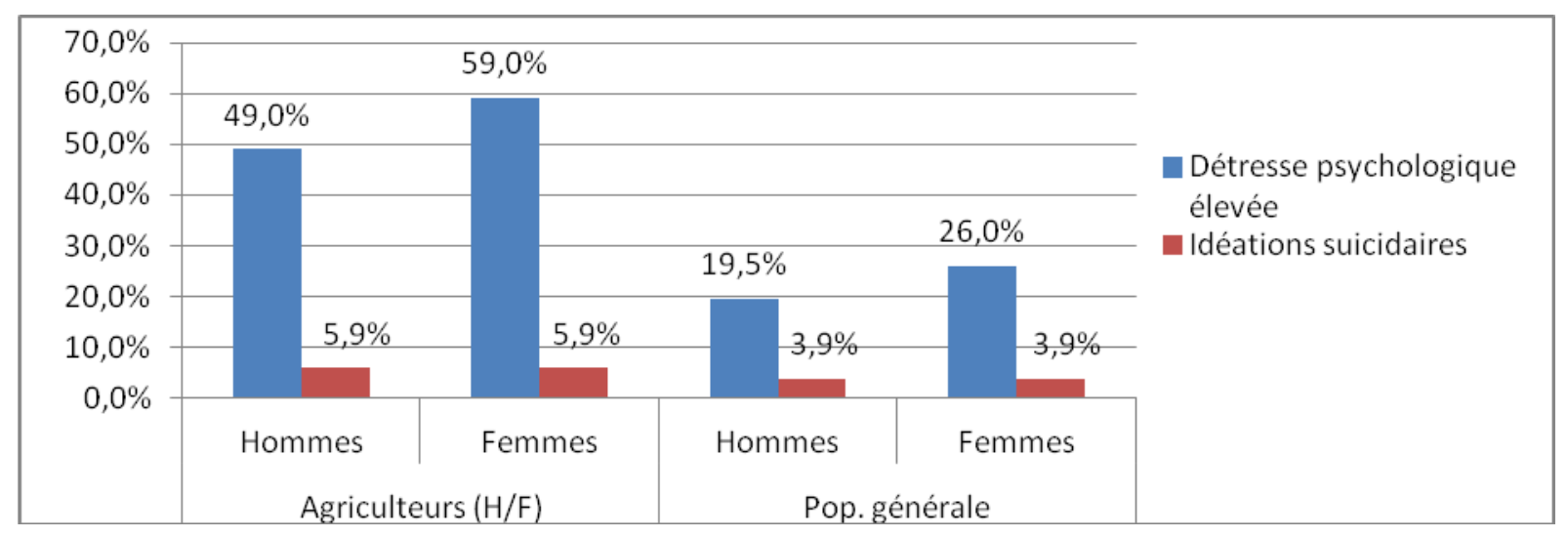

Il y a aussi d'autres problèmes de santé pour lesquels les hommes ruraux sont surreprésentés : le tabagisme, l'embonpoint, la sédentarité, les comportements à risque élevé de blessure ou pouvant causer la mort (par exemple: conduite automobile dangereuse), l'évitement de la demande d'aide, l'alimentation malsaine, le soutien social limité et la plus grande tendance à minimiser les risques (Courtenay, 2011; Judd, Jackson, Komiti et al., 2006; Martinez et al., 2004).

\section{SOUTIEN SOCIAL ET ISOLEMENT}

Un nouveau domaine de préoccupations oriente les recherches : l'expérience et l'influence du soutien social sur la santé des populations rurales. II s'agit d'une piste de recherche intéressante puisque le soutien social est identifié comme étant la principale stratégie en général et auprès des communautés rurales en particulier pour promouvoir la santé et le bienêtre (McLaren et Challis, 2009). De manière générale, les hommes ont, plus souvent que les femmes, un faible niveau de soutien social, une condition reconnue comme étant un important facteur de risque en matière de santé mentale et de suicide en particulier (Courtenay, 2011; 
Houle, 2005; Tremblay, Cloutier, Antil, Bergeron et Lapointe-Goupil, 2005). À ce jour, la plupart des études qui s'intéressent à l'influence du soutien social sur la santé psychologique des hommes ruraux ciblent les agriculteurs. La qualité du soutien social chez les agriculteurs est influencée par l'isolement, la compétition grandissante, le déclin de la coopération entre les agriculteurs et la dévitalisation des régions rurales (Lafleur et Allard, 2006; Reese, 2002, dans Sturgeon et Morrissette, 2010). La mécanisation et l'informatisation de l'agriculture diminuent les occasions de contacts directs avec les autres agriculteurs. La combinaison de ces tendances contribue à l'isolement social et à l'effritement de la solidarité.

Au Québec, une étude s'intéresse spécifiquement au soutien social chez les agriculteurs. Parent, Perrier et Rousseau (2010) révèlent que chez les agriculteurs de moins de 35 ans, $60 \%$ sont à risque d'isolement social. Parmi les jeunes agriculteurs, le groupe ayant des réseaux sociaux élargis et solides présente un faible sentiment de solitude et un éventail diversifié de soutien (amis, voisins, professionnels). Le groupe des jeunes agriculteurs plus isolés socialement est caractérisé par de petits réseaux sociaux et une tendance à restreindre le soutien social à leur réseau familial. Cela est d'autant plus vrai chez les célibataires dont près de la moitié (49\%) ont un réseau de soutien social très restreint, comparativement à $27 \%$ chez les conjoints de fait et $28 \%$ chez les mariés.

D'ailleurs, une forte majorité des célibataires (95\%) considèrent que l'agriculture est un obstacle aux rencontres amoureuses. Une étude de Ni Laoire (2001) éclaire la question du célibat des agriculteurs dans le contexte général de la mobilité sociale en milieu rural. Les personnes qui ont les plus hauts niveaux d'éducation, principalement des femmes, tendent à migrer vers les villes où elles se retrouvent majoritairement dans le secteur des services. Pour leur part, les jeunes hommes sont encouragés à demeurer dans leur communauté, motivés par des opportunités économiques dans des domaines traditionnellement masculins, soit principalement dans les secteurs primaire et secondaire, lesquels requièrent souvent peu de scolarité. Par exemple, le transfert de ferme s'effectue traditionnellement de père en fils, ce qui peut contribuer à la différence de sexe en matière de mobilité.

\section{L'UTILISATION DES SERVICES}

Les hommes ruraux utilisent moins les services de santé que les femmes rurales ou que les hommes urbains, y compris pour les problèmes de santé psychologique (Courtenay, 2011). Les hommes ruraux tendent à se débrouiller par eux-mêmes avec leurs problèmes, y compris ceux d'ordre psychologique, conformément à la norme masculine d'autonomie (Judd, Jackson, Komiti et al., 2006; Komiti, Judd et Jackson, 2006). Dans ces circonstances, les réseaux informels tendent à être privilégiés plutôt que le recours à des services de santé et de services sociaux en dehors de la communauté (Kutek et al., 2011; Lafleur et Allard, 2006; Sturgeon et Morrissette, 2010). Les hommes ruraux, et plus particulièrement les agriculteurs, entretiennent davantage des attitudes, valeurs et conceptions qui agissent comme des barrières à la demande d'aide, comme la valorisation de l'autonomie, le stoïcisme et la stigmatisation des problèmes psychologiques (Courtenay, 2011; Judd, Jackson, Fraser et al., 2006; Judd, Jackson, Komiti et al., 2006; Lafleur et Allard, 2006; Sturgeon et Morrissette, 2010). Le souci de 
confidentialité et la crainte d'être stigmatisé pour avoir utilisé les services de santé mentale en retiennent plusieurs (Fraser et al., 2005; Pugh et Cheers, 2010).

En combinant les données sur l'état de santé psychologique des hommes ruraux et leurs habitudes d'utilisation des services de santé, on constate que ce groupe peut être considéré comme étant particulièrement vulnérable. À ce portrait s'ajoutent plusieurs situations qui contribuent à marginaliser les hommes ruraux.

\section{LA STIGMATISATION ET LA MARGINALISATION DES HOMMES RURAUX}

La ruralité évoque souvent le charme bucolique des campagnes et la qualité d'un rythme de vie plus lent qu'en ville. Si le lieu peut être idéalisé, la situation est plus complexe pour les résidents ruraux, qui peuvent être stigmatisés ou marginalisés.

La stigmatisation des résidents ruraux s'observe dans certains stéréotypes qui leur sont associés. C'est un phénomène qui n'est pas récent; déjà en 1872, Marx et Engel considéraient la campagne comme un milieu d'oppression, d'ignorance et de pauvreté, où l'effet urbanisant du capitalisme devait être vu comme bénéfique (Pugh et Cheers, 2010). De nos jours, le terme redneck, associé aux résidents ruraux, porte un sens profondément péjoratif. II évoque des gens sous-éduqués, à l'accent étrange, culturellement arriérés (Bye, 2009; Little, 2006). Au Québec, on a un équivalent de cette expression dans le terme « habitant », non pas dans le sens de quelqu'un qui habite un endroit, mais plutôt en référence aux mêmes caractérisations péjoratives. De plus, cette représentation négative alimente la supposition que la vie urbaine serait plus sophistiquée, plus moderne et plus désirable que la vie rurale (Cambell, Mayerfeld Bell et Finney, 2006; Pugh et Cheers, 2010).

En Nouvelle-Zélande, les «Southern men» sont décrits comme des hommes durs, silencieux et qui travaillent dans un environnement extérieur rigoureux (Little, 2006). La description comprend aussi le manque de bonnes manières, la vulgarité et les déficits dans les habiletés sociales. Sur le plan des relations hommes-femmes, le fort taux de célibat des agriculteurs est souligné comme une problématique préoccupante. Little rapporte le point de vue de femmes néo-zélandaises selon lesquelles « l'homme du Sud a besoin d'être civilisé par l'influence d'une femme et les attributs de la masculinité ont besoin d'être domestiqués, de manière à atteindre une stabilité dans les relations de genre et dans la vie de famille » (Little, 2006, p. 199).

Plusieurs dénoncent la dévalorisation de l'agriculture, qui fait percevoir les agriculteurs comme des pollueurs ou comme des gens responsables de cruauté envers les animaux (Canadian Agricultural Safety Association, 2005; Labrecque-Duchesneau, Gagné et Picard, 2011; Lafleur et Allard, 2006; Parent et al., 2010). De plus, les différentes crises que vit l'agriculture ont amené une série de mesures sociales et de programmes de subventions visant à contrer les dégradations environnementales, les risques d'épidémies et de pandémies associés à l'élevage industriel et au contexte de mondialisation et les fluctuations du marché. Dans ce contexte, les agriculteurs sont souvent perçus comme des quémandeurs d'argent ou comme un groupe qui vit aux crochets de l'État. 
La stigmatisation des agriculteurs s'observe aussi à travers les tensions que génère la cohabitation avec les autres résidents ruraux. Le fait de travailler avec des animaux implique de longues heures de travail qu'ils enchaînent la plupart du temps sept jours par semaine. Le bruit, la circulation de la machinerie et l'odeur que dégage l'épandage de fumier sur des terres agricoles sont parmi les principaux points de tension exprimés par les résidents ruraux qui aspirent à la quiétude des campagnes (Brisson, Richardson et Gagné, 2010; LabrecqueDuchesneau et al., 2011).

En plus de la stigmatisation, les résidents ruraux doivent aussi se débattre avec une certaine marginalisation de leurs préoccupations. Malgré certaines problématiques préoccupantes, notamment la santé psychologique des hommes ruraux, les populations rurales et leurs problématiques spécifiques demeurent sous-représentées (Pugh et Cheers, 2010; Riebschleger, 2007). Par exemple, il est connu depuis longtemps au Québec que les taux de suicide sont plus élevés dans les régions rurales que dans les régions urbaines. Pourtant, rares sont les initiatives à aborder les spécificités du contexte rural, tant en recherche que dans les programmes de formation. En Australie, la situation est bien différente alors que la nouvelle politique nationale de santé des hommes reconnaît ceux qui vivent en milieu rural ou éloigné comme un groupe populationnel touché par des risques spécifiques en matière de santé et de bien-être et pour qui la recherche et les réponses sociales doivent être développées (Department of Health and Aeging of Australia, 2010). De plus, des campagnes de prévention de la dépression ciblent spécifiquement les hommes ruraux, notamment par le marketing social et par la formation des médecins ruraux (Australian Centre for Agricultural Health and Safety, 2006).

Les données présentées jusqu'ici nous amènent à nous interroger sur la trop faible préoccupation que démontrentles différentes instances responsables des services sociaux relativement à la santé psychologique des hommes ruraux. Pourtant, à plusieurs égards, les hommes ruraux forment un groupe 1) vulnérable sur le plan de la santé psychologique, 2) qui fait l'objet de stéréotypes persistants et dans le cas des agriculteurs, d'une dévalorisation de la profession et 3) dont les préoccupations sont largement marginalisées ou ignorées. Dans ce contexte, plusieurs valeurs chères au service social sont mises à mal, comme l'équité, la justice sociale et la solidarité envers les groupes marginalisés (Brodeur et Berteau, 2007). À ce jour, quelques auteurs fournissent des bases théoriques à l'étude des masculinités rurales qui peuvent faire avancer la compréhension que nous avons de la problématique.

\section{GENRE ET SANTÉ : QUELLES BASES THÉORIQUES POUR LES HOMMES RURAUX?}

Les données probantes en matière de santé et de bien-être (physique et psychologique) « convergent pour confirmer que les écarts entre les hommes et les femmes n'ont que très peu de bases physiologiques. Ils sont plutôt reliés aux effets de la socialisation masculine » (Tremblay et Déry, 2010, p. 324). C'est le cas de la tendance des hommes «à percevoir tardivement les symptômes, à moins consulter un professionnel de la santé, à moins utiliser les mesures de prévention et, pour certains, à faire preuve d'un radicalisme comportemental ou du moins à adopter plus de comportements à risque » (Tremblay et Déry, 2010, p. 311). C'est principalement pour cette raison que les sciences sociales s'intéressent de plus en plus à 
l'influence du genre (les masculinités et les féminités) sur les expériences et les pratiques de santé, et inversement, à la façon dont ces expériences et pratiques de santé constituent des manières d'agir le genre (Bottorff, Oliffe, Kelly et Chambers, 2012; Connell, 1995; Courtenay, 2011; Robertson, 2007; Tremblay et Déry, 2010). Parmi les études sur les réalités masculines, plusieurs approches théoriques permettent de mieux comprendre les interactions entre le genre et la santé, et certaines d'entre elles abordent les masculinités rurales. Dans la section qui suit, ces approches sont soumises à une analyse inspirée des travaux de Genest-Dufault et Tremblay (2010).

Le paradigme de la masculinité hégémonique, développé par Connell (1995), fait ressortir les relations de pouvoir entre les hommes et les femmes, mais aussi entre les hommes euxmêmes. Plusieurs formes de masculinité entretiennent entre elles des rapports hiérarchiques, avec au sommet, la masculinité hégémonique. Cette masculinité valorise l'antiféminité, la compétition, l'agressivité, l'hétérosexualité et l'homophobie. Par oppression ou exclusion, elle subordonne les autres formes de masculinités. Bien peu d'hommes atteignent les standards fixés par la masculinité hégémonique.

Ce paradigme est le plus utilisé dans les écrits sur les masculinités rurales (Alston et Kent, 2008; Bye, 2009; Cambell et al., 2006; Courtenay, 2011; Little, 2006; Ni-Laoire, 2001). En milieu rural, il est attendu que les « vrais » hommes demeurent stoïques face à l'adversité, qu'ils soient robustes, capables d'endurer de longues heures de travail dans des conditions météorologiques extrêmes ou des conditions de travail potentiellement dangereuses (Alston et Kent, 2008; Little, 2006). Chez les agriculteurs, même si les attributs physiques demeurent fort présents sur le plan symbolique, les habiletés entrepreneuriales et les compétences technologiques semblent occuper une plus grande importance (Courtenay, 2011). L'hétérosexualité occupe une place prépondérante dans la construction des masculinités rurales, ce qui explique l'exclusion ou les préjudices subis par plusieurs hommes gais en milieu rural (Bell, 2006; Little, 2002). En France, par exemple, l'homosexualité est moins bien acceptée par les agriculteurs que par l'ensemble de la population, dans des proportions respectives de $64 \%$ contre $77 \%$ (Ministère de l'intérieur 2007, dans Hervieu et Purseigle, 2012). Toutefois, certaines nuances s'imposent puisque des recherches soutiennent qu'être gai en milieu rural n'est pas nécessairement une expérience négative ou oppressive (Pugh et Cheers, 2010). Des associations gaies en milieu rural ou spécifiques aux agriculteurs existent en Europe, aux États-Unis et au Québec. Celles-ci visent à favoriser les rencontres et briser l'isolement.

Les écrits qui utilisent le paradigme hégémonique portent une très grande attention à l'oppression structurelle entre les hommes et les femmes et aux inégalités qui en découlent (Connell, 1995; Courtenay, 2011; Kimmel, Hearn et Connell, 2005; Little, 2006). Sans nier les expériences d'oppression, plusieurs auteurs soulignent que les réalités masculines ne peuvent être réduites aux dimensions toxiques ou déficitaires et que ce type d'analyse rend difficilement compte des aspects plus positifs que l'on trouve dans la socialisation masculine et qui peuvent servir de levier au changement social (Dulac, 2001; Genest-Dufault et Tremblay, 2010; Houle et Dufour, 2010; Macdonald, 2005; Tremblay et L'heureux, 2010). 
Le paradigme normatif de Pleck $(1981,1995)$ est aussi utilisé pour aborder les masculinités rurales. Comparé au cadre précédent, celui-ci s'intéresse davantage aux dimensions psychosociales des rôles masculins ${ }^{2}$. La masculinité correspond ici à un ensemble d'attitudes, de comportements et d'habiletés d'un groupe d'individus qui se conforment à un stéréotype et aux normes de la masculinité (Pleck, 1995). En cherchant à atteindre les standards culturels de masculinité, les rôles genrés peuvent être dysfonctionnels, traumatisants et inadéquats. Si, d'un côté, la conformité à ces normes peut représenter un risque pour la santé des hommes, la transgression de ces normes entraîne la honte et la stigmatisation, plus fortement sanctionnée chez les hommes que chez les femmes (Dulac, 2001). Ceux qui ne correspondent pas à ces normes de masculinité peuvent développer une image négative d'eux-mêmes, se sentir inférieurs ou dévalorisés (O'Neil, 2008; Pleck, 1995). Dans ce même cadre, O'Neil (2008) aborde le conflit de rôle de genre, soit l'écart entre ce que la personne considère comme étant masculin et ce qu'elle est comme personne. Il y a une corrélation étroite entre les conflits de rôle de genre et le niveau de dépression et de détresse psychologique (Tremblay, Roy, Morin, Desbiens et Bouchard, 2011).

Quelques recherches s'intéressent aux hommes ruraux à travers le paradigme normatif. C'est le cas de Levant et Habben (2003) qui expliquent que « la masculinité tend à être plus traditionnelle en milieu rural qu'en milieu urbain » (p. 171), mais qu'il ne faut pas sous-estimer néanmoins la diversité sur le plan du genre que l'on peut trouver parmi les hommes ruraux. La tendance, plus marquée chez les hommes ruraux que chez les hommes urbains, à moins consulter les professionnels de la santé est expliquée ici par le conflit entre les éléments de base de la psychothérapie (dévoilement de la vulnérabilité, expression des émotions) et l'idéologie de masculinité traditionnelle (stoïcisme, démonstration de la force). Ceci est amplifié par la grande visibilité sociale des individus propre aux communautés rurales.

Dans sa forme générale, la critique du paradigme normatif rejoint celle formulée à l'endroit du paradigme de la masculinité hégémonique, c'est-à-dire qu'il cible essentiellement les éléments négatifs résultant de la contrainte qu'exige cette forme de socialisation masculine. Nous ne remettons pas en question les conséquences négatives de cette socialisation sur la santé et le bien-être des hommes, mais ce paradigme occulte ou néglige les comportements positifs des hommes. Cela dit, ce paradigme est très éclairant puisque sa vision psychosociale permet de bien saisir l'interaction entre le genre et la santé. Dans son application aux masculinités rurales, nous retenons l'importance de ne pas négliger la diversité des formes de masculinité que l'on retrouve en milieu rural. Mais encore ici, une attention plus soutenue doit être accordée aux éléments positifs qu'implique la socialisation masculine chez les hommes ruraux. Les agriculteurs, par exemple, sont généralement perçus comme étant des gens courageux, vaillants et débrouillards. Positive apparaît également la solidarité entre hommes qui s'exprime, entre autres, dans le syndicalisme agricole et dans l'investissement communautaire.

2. Bien qu'il ne semble pas y avoir de définition claire du ou des rôles de genre, on peut penser que l'adhésion au rôle masculin signifie la conformité à l'idéologie masculine traditionnelle. Dans son sens pluriel, s'il en est un, l'auteur indique que « les rôles de genre sont opérationnellement définis selon les stéréotypes et les normes de rôle de genre » (Pleck, 1995, p. 12). 
Force est de constater qu'il faut un éclairage additionnel pour aborder les forces et les capacités des hommes ruraux. En ce sens, l'approche «salutogène » se propose de porter attention non seulement aux éléments préjudiciables ou aux lacunes, mais aussi aux éléments sains qui contribuent au bien-être des personnes, comme les forces et les capacités des individus et des collectivités (Macdonald, 2005). Elle tranche donc avec une certaine vision des hommes, élaborée par le passé, comme un groupe homogène et problématique (Crawshaw, 2009). À plusieurs égards, l'approche salutogène rejoint la psychologie positive (Kiselica, 2010) et l'approche centrée sur les forces, une approche de base en service social (Rapp et Goscha, 2006). Ces manières de penser la recherche et l'intervention auprès des clientèles masculines sont cohérentes avec la position de l'Organisation mondiale de la santé, qui suggère d'élargir la définition de la santé psychologique au-delà de l'étude des maladies psychologiques (Herrman et Jane-Llopis, 2005). Dans cette approche, la norme masculine de l'indépendance peut être interprétée positivement lorsqu'elle implique une indépendance vis-à-vis des stéréotypes masculins et permet de considérer la demande d'aide comme une preuve de courage (Hammer et Good, 2010). Similairement, Houle et Dufour (2010) suggèrent d'intervenir auprès des hommes suicidaires en utilisant une imagerie masculine et en faisant ressortir les contradictions entre les valeurs masculines de la personne, comme le sens du devoir, et les douloureuses conséquences sur les proches que pourrait avoir le suicide.

Ainsi, ce n'est pas tant l'application des normes masculines qui est problématique, mais plutôt la conformité rigide à ces normes et lorsque celles-ci dissuadent de s'engager dans des masculinités non traditionnelles (Kiselica, 2010; Tremblay et L'heureux, 2010). Bye (2009) présente une étude qui va au-delà de la vision déficitaire et négative des hommes ruraux et fait ressortir l'évolution et la diversité des pratiques masculines. Cette étude norvégienne constate que les jeunes hommes ruraux développent une identité masculine plus flexible et moins basée sur le travail que la génération précédente. Par exemple, ces jeunes hommes expriment une plus grande ouverture quant à la possibilité pour des hommes de choisir des professions traditionnellement féminines, et de devenir infirmiers, employés de garderie ou de travailler dans le secteur des services. L'engagement paternel joue également un grand rôle dans la construction de leur identité masculine. Cela s'explique à la fois par «les politiques sociales, par les attentes des jeunes femmes et par leur propre réflexion sur l'égalité de genre » (Bye, 2009 , p. 286). Les résultats de cette étude auraient été tout autres si l'auteure avait adopté une perspective qui focalise sur les aspects déficitaires et qui occulte les aspects positifs des pratiques masculines. Une perspective plus nuancée permet donc de mieux apprécier la diversité des pratiques masculines.

\section{Pistes de RECHERChe}

Bien que les données probantes révèlent l'urgence de se préoccuper de la santé psychologique des hommes ruraux, les préoccupations de ce groupe demeurent négligées. Nul doute que des services sociaux sont bel et bien dispensés dans les milieux ruraux à travers le Québec. Mais les pratiques des travailleurs sociaux en milieu rural demeurent méconnues. Le concept de travailleur de rang, similaire au travailleur de rue, vient de faire son apparition dans la littérature clinique (Labrecque-Duchesneau et al., 2011). Ce dernier vise l'intégration des intervenants dans le milieu rural et agricole et l'adoption d'une approche proactive auprès de la clientèle 
cible. À l'exception de cet exemple récent, les publications scientifiques québécoises sur l'intervention psychosociale auprès des hommes ruraux sont quasi inexistantes. Le même constat s'applique sur le plan de la formation et des politiques publiques : cette clientèle est absente dans ses spécificités. Cette absence contribue à maintenir les hommes ruraux dans une position marginalisée. Considérant qu'environ $20 \%$ de la population québécoise et canadienne vit en milieu rural, il semblerait légitime qu'on s'attache à mieux connaître les conditions de vie de ces communautés (Martinez et al., 2004).

Sur un plan plus positif, même si les hommes ruraux sont confrontés à de grands défis sur les plans social et sanitaire, il faudrait mieux connaître leurs forces et leurs capacités, en tant que facteurs de protection potentiels. Jusqu'ici, plusieurs études sur le milieu rural se sont penchées sur le sentiment d'appartenance et le soutien social, mais elles abordent peu la dimension de genre, pourtant centrale à l'expérience de la santé et du bien-être (Judd, Jackson, Fraser et al., 2006; Kutek et al., 2011; McLaren et Challis, 2009; Parent et al., 2010). Il serait donc intéressant de mieux connaître les rôles masculins qui soutiennent les pratiques positives de santé chez les hommes ruraux.

\section{Conclusion}

Cet article rapporte l'état des connaissances sur la santé psychologique chez les hommes ruraux et ses liens avec la construction du genre masculin. Nous avons exposé les éléments qui contribuent à faire considérer les hommes ruraux, bien que très diversifiés, comme un groupe vulnérable sur le plan de la santé psychologique, notamment en matière de suicide, d'isolement social et d'utilisation des services. À plusieurs égards, les hommes ruraux sont marginalisés par différents stéréotypes et par la négligence de leurs préoccupations par les milieux politique, académique et professionnel.

Les études sur la santé des hommes ruraux abordent rarement la dimension de genre et inversement, les études sur la santé des hommes qui prennent en compte la dimension de genre abordent rarement les spécificités du contexte rural. En partant des données probantes sur la santé psychologique des hommes ruraux, il est clair que les connaissances doivent être approfondies concernant l'interaction entre la construction de genre et les pratiques de santé. Enfin, il serait important de développer au sein du service social un domaine spécifiquement dédié à la pratique en milieu rural, comme c'est le cas notamment en Australie et aux ÉtatsUnis. Ceci permettrait de mieux connaître les initiatives locales ou régionales, les expériences novatrices, et de définir les meilleures pratiques.

Philippe Roy MA Sociologie, Doctorant en service social Université Laval

Gilles Tremblay

PhD. sciences biomédicales, t.s. Professeur titulaire Université Laval 


\section{RÉFÉRENCES}

Alston, M. et J. Kent (2008). « The Big Dry: The link between rural masculinities and poor health outcomes for farming men », Journal of Sociology, vol. 44, n² 2, p. 133-147.

Australian Centre for Agricultural Health and Safety. (2006). Rural Mental Health Blueprint a National First, Moree (Austr.), Australian Centre for Agricultural Health and Safety.

Bell, D. (2006). «Cowboy love », dans H. Cambell, M. Mayerfeld Bell et M. Finney (dir.), Country Boys: Masculinity and Rural Life, University Park (Penn.), The Pennsylvania State University Press, p. 163-182.

Booth, N., M. Briscoe et R. Powell (2000). « Suicide in the farming community: Methods used and contact with health services », Occupational and Environmental Medicine, vol. 57, p. 642-644.

Bottorff, J.L., J. Oliffe, M.T. Kelly et N.A. Chambers (2012). « Approaches to examining gender relations in health research », dans J. Oliffe et L. Greaves (dir.), Designing and conducting gender, sex and health research, Thousand Oaks (Calif.), Sage, p. 175-188.

Brisson, G., M. Richardson et D. Gagné (2010). Relation entre l'agriculture et la qualité de vie des communautés rurales et périurbaines : synthèse Politiques publiques et santé, Québec, Institut national de santé publique du Québec.

Brodeur, N. et G. Berteau (2007). «La réflexion éthique : une dimension essentielle dans la pratique du travail social », dans J.-P. Deslauriers et Y. Hurtubise (dir.), Introduction au travail social, Québec, Presses de l'Université Laval, p. 241-266.

Butler, J. (2005). Trouble dans le genre. Pour un féminisme de la subversion, Paris, La Découverte.

Bye, L.M. (2009). «"How to be a rural man": Young men's performances and negotiations of rural masculinities », Journal of Rural Studies, vol. 25, $n^{\circ} 3$, p. 278-288.

Cambell, H., M. Mayerfeld Bell et M. Finney (2006). Country Boys: Masculinity and Rural Life, University Park (Penn.), The Pennsylvania State University Press.

Camirand, H. et V. Nahnou (2008). La détresse psychologique chez les Québécois en 2005, Québec: Institut de la statistique du Québec.

Canadian Agricultural Safety Association (2005). National stress and mental survey of Canadian farmers, Winnipeg, Canadian Agricultural Safety Association - CASA.

Collier, K. (2006). Social work with rural peoples (3éd.), Vancouver, New Star Books.

Connell, R.W. (1995). Masculinities, Berkeley et Los Angeles, University of California Press.

Courtenay, W.H. (2011). Dying to be men, New York, Routledge. 
Crawshaw, P. (2009). «Critical perspectives on the health of men: Lessons from medical sociology », Critical Public Health, vol. 19, p. 279-285.

Department of Health and Aeging of Australia. (2010). Developing a Men's Health Policy for Australia. Australian Government.

[http://www.health.gov.au/internet/main/publishing.nsf/Content/9299CC85891AB2B6CA25745F0023D0E3/\$ File/mens-developing.pdf.]

Dulac, G. (2001). Aider les hommes... aussi, Montréal, VLB éditeur.

Farley, O.W. (1982). Rural social work practice, New York, Free Press.

Fraser, C., F. Judd, H. Jackson, G. Murray, J.S. Humphreys et G. Hodgins (2002). « Does one size really fits all? Why the mental health of rural Australians requires further research », Australian Journal of Rural Health, vol. 10, p. 288-295.

Fraser, C.E., K.B. Smith, F. Judd, J.S. Humphreys, L.J. Fragar et A. Henderson (2005). « Farming and mental health problems and mental illness », International Journal of Social Psychiatry, vol. 51, $\mathrm{n}^{\circ}$ 4, p. 340-349.

Gagné, M., G. Légaré, P.A. Perron et D. St-Laurent (2011). La mortalité par suicide au Québec: données récentes de 2005 à 2009. Mise à jour 2011. Québec: Institut national de santé publique du Québec.

Genest-Dufault, S. et G. Tremblay (2010). « Cinq paradigmes compréhensifs des hommes et des masculinités: proposition d'une classification originale », dans J.-M. Deslauriers, G. Tremblay, S. Genest-Dufault, D. Blanchette et J.-Y. Desgagnés (dir.), Regards sur les hommes et les masculinités, Québec, Presses de l'Université Laval, p. 61-90.

Hammer, J.H. et G.E. Good (2010). «Positive psychology: An empirical examination of beneficial aspects of endorsement of masculine norms », Psychology of Men \& Masculinity, vol. $11, n^{\circ} 4$, p. 303-318.

Herrman, H. et E. Jane-Llopis (2005). « Mental health promotion in public health », International Journal of Health Promotion and Education, Suppl. 2, p. 42-47, 63, 69.

Hervieu, B. et F. Purseigle (2012). «Les mondes agricoles: une minorité éclatée, ancrée à droite », Les électorats sociologiques (Toulouse, CEVIPOF), vol. 13, février.

Houle, J. (2005). La demande d'aide, le soutien social et le rôle masculin chez des hommes qui ont fait une tentative de suicide, thèse de doctorat, Université du Québec à Montréal, Montréal.

[http://accesbib.uqam.ca/cgi-bin/bduqam/transit.pl?\&noMan=24065952]

Houle, J. et M.-A. Dufour (2010). «Intervenir auprès des hommes suicidaires », Psychologie Québec, vol. 27, $\mathrm{n}^{\circ} 1$, p. 27-29.

Institut canadien d'information sur la santé (2006). Comment se portent les Canadiens vivant en milieu rural, Ottawa, Institut canadien d'information sur la santé. 
Judd, F., H. Jackson, C. Fraser, G. Murray, G. Robins et A. Komiti (2006). « Understanding suicide in Australian farmers », Social Psychiatry and Psychiatric Epidemiology, vol. 41, $\mathrm{n}^{\circ} 1$, p. 1-10.

Judd, F., H. Jackson, A. Komiti, G. Murray, C. Fraser, A. Grieve, et al. (2006). « Help-seeking by rural residents for mental health problems: The importance of agrarian values », Australian and New Zealand Journal of Psychiatry, vol. 40, nº 9, p. 769-776.

Kimmel, M.S., J. Hearn et R.W. Connell (2005). Handbook of studies on men \& masculinities, Thousand Oaks (Calif.), Sage Publications.

Kiselica, M.S. (2010). «Promoting positive masculinity while addressing gender role conflict: A balanced theoretical approach to clinical work with boys and men », dans C. Blazina et D.S. Shen-Miller (dir.), An international psychology of men: Theoretical advances, case studies, and clinical innovations, New York, Routledge et Taylor \& Francis Group, p. 127-156.

Komiti, A., F. Judd et H. Jackson (2006). «The influence of stigma and attitudes on seeking help from a GP for mental health problems: A rural context », Social Psychiatry and Psychiatric Epidemiology, vol. 41, nº 9, p. 738-745.

Kutek, S., D. Turnbull et A.-K. Fairweather-Schmidt (2011). « Rural men's subjective well-being and the role of social support and sense of community: Evidence for the potential benefit of enhancing informal networks », Australian Journal of Rural Health, vol. 19, n 1, p. 20-26.

Labrecque-Duchesneau, M., J. Gagné et F. Picard (2011). « Le travailleur de rang : une plusvalue pour une qualité de vie en milieu rural », Intervention, vol. 135, n² 2, p. 106-114.

Lafleur, G. et M.-A. Allard (2006). Enquête sur la santé psychologique des producteurs agricoles du Québec, COOP Fédérée.

Levant, R.F. et C. Habben (2003). «The new psychology of men: Application to rural men », dans B.H. Stamm (dir.), Rural behavioral health care: An interdisciplinary guide, Washington (D. C.), American Psychological Association, p. 171-180.

Lindsay, J., G. Rondeau et J.-Y. Desgagnés (2010). « Bilan et perspectives du mouvement social des hommes au Québec entre 1975 et 2010 ». dans J.-M. Deslauriers, G. Tremblay, S. Genest-Dufault, D. Blanchette et J.-Y. Desgagnés (dir..), Regards sur les hommes et les masculinités, Québec, Presses de l'Université Laval, p. 13-43.

Little, J. (2002). Gender and rural geography: Identity, sexuality and power in the countryside, Harlow et New York, Prentice Hall.

Little, J. (2006). « Embodiement and Rural Masculinity », dans H. Cambell, M. Mayerfeld Bell et M. Finney (dir.), Country boys: Masculinity and rural life, University Park (Penn.), Pennsylvania State University Press, p. 183-202.

Lohmann, N. et R.A. Lohmann (2005). Rural social work practice, New York, Columbia University Press.

Macdonald, J.J. (2005). Environments for health: A salutogenic approach, Londres et Sterling (Virginie), Earthscan. 
Martinez-Brawley, E.E. (1999). Close to home: Human services and the small community, Washington (D. C.), NASW Press.

Martinez, J., R. Pampalon, D. Hamel et G. Raymond (2004). Vivre dans une collectivité rurale plutôt qu'en ville fait-il vraiment une différence en matière de santé et de bien-être?, Québec, Institut national de santé publique du Québec.

McLaren, S. et C. Challis (2009). «Resilience among men farmers: The protective roles of social support and sense of belonging in the depression-suicidal ideation relation », Death Studies, vol. 33, $\mathrm{n}^{0} 3$, p. 262-276.

Miller, K. et C. Burns (2008). « Suicides on farms in South Australia, 1997-2001 », Australian Journal of Rural Health, vol. 16, p. 327-331.

Ni Laoire, C. (2001). «A matter of life and death? Men, masculinities and staying "behind" in rural Ireland », Sociologia Ruralis, vol. 41, $\mathrm{n}^{\circ} 2$, p. 220-236.

O'Neil, J.M. (2008). « Summarizing 25 years of research on men's gender role conflict using the Gender Role Conflict Scale: New research paradigms and clinical implications », The Counseling Psychologist, vol. 36, n 3, p. 358-445.

Parent, D., J.-P. Perrier et G. Rousseau (2010). Analyse de l'isolement social, de la sociabilité et de la qualité du soutien social chez les jeunes agriculteurs québécois, Québec, Université Laval, Traget Laval.

Pickett, W., W.D. King, T. Faelker, R. Lees, H. Morrison et M. Bienefeld (2000). Le suicide chez les exploitants agricoles canadiens, Ottawa, Agence de santé du Canada.

Pleck, J.H. (1981). The myth of masculinity, Cambridge, MIT Press.

Pleck, J.H. (1995). « The gender role strain paradigm: An update », dans R.F. Levant et W.S. Pollack (dir.), A new psychology of men, New York, Basic Books, p. 11-32.

Préville, M., R. Boyer, R. Hébert, G. Bravo et M. Seguin (2005). « Correlates of suicide in the older adult population in Quebec », Suicide Life Threat Behavior, vol. 35, n 1, p. 91-105.

Pugh, R. et B. Cheers (2010). Rural Social Work: An international perspective, Bristol, Policy Press.

Rapp, C.A. et R.J. Goscha (2006). The strengths model: Case management with people with psychiatric disabilities ( ${ }^{\mathrm{e}}$ éd.), Oxford et Toronto, Oxford University Press.

Riebschleger, J. (2007). «Social worker's suggestions for effective rural practice », Families in Society, vol. 88, p. 203-213.

Robertson, S. (2007). Understanding Men and Health: Masculinities, Identity and Well-being. Breshire, Open University Press.

St-Laurent, D. et M. Gagné (2008). Surveillance de la mortalité par suicide au Québec. Ampleur et évolution du problème de 1981 à 2006, Institut national de santé publique du Québec.

[http://collections.banq.qc.ca/ark:/52327/1764676] 
Statistique Canada (2006). Recensement de l'agriculture, Gouvernement du Canada. [http://www.statcan.gc.ca/ca-ra2006/index-fra.htm.]

Sturgeon, R. et P.J. Morrissette (2010). «A qualitative analysis of suicide ideation among Manitoban farmers », Canadian Journal of Counselling and Psychotherapy, vol. 44, $\mathrm{n}^{\circ} 2$, p. 191-207.

Tremblay, G., R. Cloutier, T. Antil, M.-È. Bergeron et R. Lapointe-Goupil (2005). La santé des hommes: portrait de la situation des hommes en matière de santé au Québec, Québec, Ministère de la Santé et des Services sociaux.

Tremblay, G. et F. Déry (2010). «La santé des hommes au Québec », dans J.-M. Deslauriers, G. Tremblay, S. Genest-Dufault, D. Blanchette et J.-Y. Desgagnés (dir.), Regards sur les hommes et les masculinités, Québec, Les Presses de l'Université Laval, p. 305-330.

Tremblay, G. et P. L'Heureux (2010). « La genèse de la construction de l'identité masculine », dans J.-M. Deslauriers, G. Tremblay, S. Genest-Dufault, D. Blanchette et J.-Y. Desgagnés (dir.), Regards sur les hommes et les masculinités, Québec, Les Presses de l'Université Laval, p. 91-124.

Tremblay, G., P. Roy, M.-A. Morin, V. Desbiens et P. Bouchard (2011). « Conflits de rôle de genre et dépression chez les hommes », Revue québécoise de psychologie, vol. 32, $\mathrm{n}^{\circ} 1$, p. 181-200. 